\title{
Diseño de cuestionarios de frecuencia de consumo para estudiar la relación dieta-cáncer en Colombia
}

\author{
Oscar F. Herrán ${ }^{1,2}$, María F. Ardila², Martha P. Rojas³ ${ }^{3}$ Gustavo A. Hernández
}

\author{
1 Escuela de Nutrición y Dietética, Universidad Industrial de Santander, Bucaramanga, Colombia \\ 2 Grupo Observatorio Epidemiológico de Enfermedades Cardiovasculares, Centro de Investigaciones \\ Epidemiológicas, Universidad Industrial de Santander, Bucaramanga, Colombia \\ ${ }^{3}$ Grupo Investigación Epidemiológica del Cáncer, Instituto Nacional de Cancerología, Bogotá, D.C., Colombia
}

Introducción. En Colombia, el cáncer es la tercera causa de muerte en población adulta. A la dieta se le atribuye el 35\% de las muertes por cáncer y la prevención del $90 \%$ del cáncer de colon y recto.

Objetivo. Desarrollar cinco cuestionarios de frecuencia de consumo para estudiar la relación entre dieta y cáncer, en cinco ciudades colombianas (Barranquilla, Cartagena, Santa Marta, Bogotá y Bucaramanga y su área metropolitana), a partir de recordatorios de 24 horas recolectados en la Encuesta Nacional de la Situación Nutricional, 2005.

Materiales y métodos. Cada cuestionario involucró seis etapas en su desarrollo y tiene tres secciones. La lista de chequeo se obtuvo para representar trece nutrientes mediante el método de la maximización de la varianza (Max $r$ (B) y fue complementada con nueve categorías de frecuencia de consumo en el último año. Las dos secciones complementarias indagan sobre el consumo de alimentos, el número de comidas al día y las formas de preparación, relacionadas con el desarrollo de cáncer.

Resultados. Entre 22 y 32 alimentos fueron discriminados e incluidos en los cuestionarios. Sólo nueve alimentos fueron comunes en las listas de chequeo. Nueve de los 13 nutrientes están representados con $\mathrm{R}^{2}$ superiores a 0,80 , mínimo 0,47 y máximo 1,0 .

Conclusiones. Por primera vez en Colombia se desarrollaron cuestionarios específicos para estudiar la relación dieta-cáncer. Las listas de chequeo son exhaustivas, incorporaron la variabilidad entre personas, los hábitos y la cultura alimentaria. Se proponen nueve alternativas de análisis para los cuestionarios. Los cuestionarios son útiles en la investigación epidemiológica, y los resultados pueden calibrarse con estudios de reproducibilidad y validez.

Palabras clave: dieta, epidemiología nutricional, neoplasias, transición nutricional, Colombia.

Design of dietary questionnaires to study the relationships between diet and cancer prevalence in Colombia

Introduction. In Colombia, cancer ranks third as a cause of death in the adult population. Thirty-five percent of cancer deaths are attributable to dietary factors and that $90 \%$ of colorectal cancers can be prevented by appropriate diets.

Objective. A dietary questionnaire was developed to study the relationship between diet and cancer for five cities in Colombia (Barranquilla, Cartagena, Santa Marta, Bogotá and Bucaramanga and its metropolitan area), based on 24-hour dietary survey data accumulated by the National Survey of Nutritional Status-2005.

Material and methods. Each questionnaire had 3 sections and involved six stages in its development. A checklist representing thirteen nutrients was formulated by means of the method of the variance maximization (Max_r $\mathbb{B}$ ); it was supplemented with nine categories of consumption frequency. The two complementary sections inquired about the consumption of food, number of meals per day, and methods of preparation, related with cancer development.

Results. Between 22 and 32 foods were discriminated for each questionnaire. Only nine foods were common in the checklists of each of the five cities. Nine of the thirteen nutrients are represented with correlations higher than 0.80 , with a minimum 0.47 and a maximum of 1.0 .

Conclusions. For the first time in Colombia specific questionnaires were developed to study the dietcancer relationship. The checklist was comprehensive and it incorporated between-person variability, as well as the habits and food culture of each city. We propose nine analysis alternatives for the questionnaire. The questionnaires will prove useful in epidemiological research, although they require calibration with studies of reproducibility and validity.

Key words: diet, nutritional epidemiology, neoplasms, nutritional transition, Colombia. 
En Colombia el cáncer es la tercera causa de muerte, antecedida sólo por las del sistema circulatorio y las atribuidas a causas externas $(1,2)$. En el 2005, murieron 30.717 personas por tumores malignos; la mortalidad por cáncer representó el $16,3 \%$ del total de defunciones y alcanzó una tasa de 69,9 por 100.000 habitantes $(1,3,4)$. A diferencia de lo que ocurre en los países desarrollados, con excepción del cáncer de estómago, los otros tipos siguen en aumento o no se reducen de manera significativa $(5,6)$.

El cáncer es una enfermedad multifactorial que ocurre debido a la interacción poco comprendida entre factores ambientales y genéticos; la dieta es un factor ambiental al que se atribuye, al menos, $35 \%$ de las muertes por cáncer en general $(7,8)$. La dieta es una exposición difícil de medir por la gran variabilidad en ella y las complejas interrelaciones entre los nutrientes (9-11); su correcta medición es un reto en la investigación epidemiológica.

Para estudiar la relación entre cáncer y dieta, se han usado tradicionalmente cuestionarios de frecuencia de consumo. Sin embargo, uno de éstos, desarrollado en una sociedad y cultura en particular, puede que no sea útil en otra $(12,13)$, lo que obliga al desarrollo de cuestionarios específicos, y a realizar estudios de reproducibilidad y validez complementarios que permitan calibrar los resultados de la investigación epidemiológica (14-17).

Los resultados contradictorios sobre la relación entre dieta y cáncer, en buena medida, son consecuencia de dietas heterogéneas, de la poca especificidad y validez de los cuestionarios de frecuencia de consumo utilizados para medirla o de análisis estadísticos limitados o erróneos (14-22).

Dadas las relaciones entre dieta y cáncer, es necesario desarrollar métodos para medir correctamente esta exposición. La medición incorrecta se traduce en atenuación de las medidas epidemiológicas. Los cuestionarios de frecuencia de consumo son el método simplificado más utilizado para la medición de la exposición de la dieta a largo plazo. La elección de la lista

\section{Correspondencia:}

Oscar Fernando Herrán, Centro de Investigaciones Epidemiológicas, Facultad de Salud, Universidad Industrial de Santander, Carrera 32 № 29-31, tercer piso, oficina 304, Bucaramanga, Colombia.

Telefax: (577) 6345781

herran@uis.edu.co y oscar.herran@gmail.com

Recibido: 03/06/09; aceptado:08/11/09 de chequeo de alimentos es el primer paso en la construcción del cuestionario; de la elección correcta de los alimentos dependen los resultados (9-13).

Recientemente, se realizó la Encuesta Nacional de la Situación Nutricional, 2005 (ENSIN-2005) que, con base en recordatorios del consumo de las últimas 24 horas (R24H), determinó la ingesta usual y la prevalencia de déficit de consumo de nutrientes por regiones y departamentos del país (23).

El objetivo fue desarrollar cinco cuestionarios de frecuenciadeconsumo-unoporciudad-específicos para el estudio de la relación entre dieta y cáncer, a partir de la información recolectada en la ENSIN2005 y con base en la información acumulada, empleando para ello métodos estadísticos avanzados y computacionales intensivos. Además, se discuten las ventajas y limitaciones de estos cuestionarios de frecuencia de consumo y la manera de utilizar la información derivada de ellos, para establecer adecuadamente la relación entre dieta y cáncer.

\section{Materiales y métodos}

Cada cuestionario de frecuencia de consumo contempló el desarrollo de seis etapas:

1) selección de ciudades,

2) determinación de la muestra e información sobre consumo de alimentos,

3) derivación de la lista de chequeo,

4) definición de categorías de frecuencia de consumo y tamaños de porción,

5) definición de alimentos 0 preparaciones asociadas como factores de riesgo o protectores en la relación dieta-cáncer y

6) definición de formas de cocción descritas como factor de riesgo o protector en esta relación.

Como resultado, cada cuestionario de frecuencia de consumo se diseñó con tres secciones. La primera incorporó la lista de chequeo que es específica para cada ciudad; la segunda una lista de alimentos definida a priori, y la última, preguntas específicas sobre la forma habitual de cocción de algunos alimentos. Las dos últimas secciones son comunes a todos los cuestionarios de frecuencia de consumo.

\section{Selección de ciudades}

Se seleccionaron cinco ciudades: Barranquilla, Cartagena, Santa Marta, Bogotá y Bucaramanga y 
su área metropolitana; las tres primeras localizadas en la zona norte de Colombia y con cultura alimentaria caribe, y las otras dos correspondientes una a la capital del país y la otra a la ciudad más desarrollada del nororiente colombiano, ambas con cultura alimentaria andina.

Estas ciudades fueron seleccionadas de manera estratégica porque, además de presentar diferencias en el grado de desarrollo social y económico, y en las tasas de cánceres relacionados con la dieta, exhiben características particulares de la alimentación que permiten discriminar a los sujetos por su dieta.

\section{Muestra e información sobre consumo de alimentos}

Los $\mathrm{R} 24 \mathrm{H}$ que sirvieron como insumo para la derivación de la lista de chequeo de los cuestionarios de frecuencia de consumo, se obtuvieron en medio magnético de las bases de datos de la ENSIN2005, suministradas por el Instituto Colombiano de Bienestar Familiar (23).

La ENSIN-2005 se realizó en una submuestra de 17.740 hogares que sirvió para la Encuesta Nacional de Demografía y Salud, 2005; su universo fue el $99 \%$ de la población urbana y rural de 32 departamentos y de la capital de Colombia. Los $\mathrm{R} 24 \mathrm{H}$ fueron recolectados en 39.413 sujetos y a $9 \%$ de ellos se les tomó un segundo $\mathrm{R} 24 \mathrm{H}$ en un día no consecutivo del primero, con el fin de obtener su ingesta usual. La información recolectada sobre consumo en la ENSIN-2005 se caracteriza por el alto grado de precisión lograda en la determinación de los tamaños de porción. Este estudio no hizo ninguna medición complementaria.

La metodología que describe el diseño y selección de la muestra en la ENSIN-2005, así como el proceso que permitió recolectar los $\mathrm{R} 24 \mathrm{H}$, codificarlos, digitarlos y validar su información, está descrita en el reporte técnico publicado (24).

Los sujetos residentes en cada ciudad de interés se seleccionaron mediante el código asignado por el Departamento Administrativo Nacional de Estadísticas (DANE) de las bases con la información de consumo, limitando la edad de los mismos entre los 30 y los 75 años, dado que es en ese grupo etario en el que se aplicarán los cuestionarios de frecuencia de consumo en futuros estudios epidemiológicos.

Se utilizó el total de la información disponible en la ENSIN-2005 por ciudad, dados los anteriores criterios; 842 sujetos aportaron información en $906 \mathrm{R} 24 \mathrm{H}$. Para derivar la lista de chequeo de cada cuestionario de frecuencia de consumo, se analizó la información de 154 sujetos residentes en Barranquilla, 344 en Bogotá, 115 en Bucaramanga, 130 en Cartagena y 99 en Santa Marta.

\section{Primera sección del cuestionario de frecuencia de consumo}

Derivación de la lista de chequeo. Como lo sugieren Willet y Margetts, varios principios guiaron la conformación de la lista de chequeo $(9,10)$ : que tuviera poder discriminador entre sujetos por la ingestión de los principales nutrientes relacionados con el desarrollo de cáncer (energía total, proteínas, carbohidratos, grasa total, grasa saturada, grasa monoinsaturada, grasa poliinsaturada, fibra soluble e insoluble de la dieta, hierro, vitamina A-carotenos-, vitamina $\mathrm{C}$, ácido fólico); que fuera limitada en alimentos, y, además, específica para cada ciudad al incorporar el hábito y el patrón de consumo.

Para lograr lo anterior, se seleccionaron los cinco primeros alimentos que contribuyeron a la variación entre personas, es decir, con mayor aporte al $R^{2}$ en un procedimiento clásico de selección por pasos y prospectiva (stepwise-forward), en el que la variable dependiente fue la ingestión total del nutriente de interés y, las que explicaran las cantidades consumidas del nutriente en cada alimento. Para que un alimento fuera elegible debía contribuir, al menos, con $2 \%$ a la ingestión total del nutriente y ser consumido por cinco o más sujetos en la respectiva ciudad.

La elección de los cinco alimentos que más contribuyen a la variabilidad aportó la capacidad discriminadora en la lista de chequeo, lo cual se logró utilizando el método de maximización del coeficiente $r$ de Pearson, conocido como Max $r(25,26)$. Este método, desarrollado en 1996 $(9,25,26)$, opera en la práctica como una regresión lineal múltiple (stepwise) $(27,28)$, pero a diferencia de ésta, el método de computación es más intensivo y tiene como objetivo establecer todos los posibles grupos de alimentos que pueden conformarse con las variables independientes (Wi), para calcular entre cada uno de ellos y la ingestión total del nutriente ( $\mathrm{Zi})$-dependiente de la variable-, un coeficiente de correlación de Pearson ( $r$ ).

Max $r$, de manera similar a la regresión lineal, estima un coeficiente de determinación $\left(R^{2} w\right)$, en el que a diferencia con el $\mathrm{R}^{2}$ tradicionalmente calculado, el subrogado Zi-Z -la ingestión total del nutriente 
de un individuo, menos la ingestión promedio del nutriente para la población-, es reemplazado por Wi-W -la ingesta del subconjunto para el individuo, menos la ingestión promedio del subconjunta en toda la población- (25). En términos estadísticos, mientras la regresión lineal maximiza la varianza explicada entre individuos, Max $r$ maximiza la correlación r de Pearson (25).

Willet propone este método y lo asimila al clásico de selección mediante regresión lineal múltiple (9); la experiencia local ha demostrado que Max $r$ es muy superior al estimar la contribución de cada ítem al $R^{2}(12,13,29,30)$. Puesto que existe evidencia de que los $\mathrm{R} 24 \mathrm{H}$ de un día no se correlacionan con los de otro consecutivo (30) y que, además, la ENSIN-2005 encuestó en días no consecutivos (23), los resultados en las listas de chequeo no están influidos por los $64 \mathrm{R} 24 \mathrm{H}$ duplicados (906 $\mathrm{R} 24 \mathrm{H}-824 \mathrm{R} 24 \mathrm{H}$ ).

Definición de categorías de frecuencia de consumo y tamaños de porción. Cada una de las cinco listas de chequeo se complementó con nueve categorías de frecuencia de consumo en el último año. Estas categorías complementarias y mutuamente excluyentes van desde dos o más veces al día hasta nunca. Además, se estimaron para cada ciudad los tamaños medios de las porciones consumidas de cada alimento seleccionado (media aritmética y geométrica), lo que permitirá traducir la lista de chequeo para estimar la ingestión de nutrientes.

\section{Segunda sección del cuestionario de frecuencia de consumo}

Definición de alimentos o preparaciones asociados como factores de riesgo o protectores en la relación dieta-cáncer. Estos alimentos se definieron después de revisar la información existente sobre la relación dieta-cáncer. Para hacer concordante esta sección con el chequeo, se preguntó por la ingestió usual en el último año $(7,8,11,31)$.

\section{Tercera sección del cuestionario de frecuencia de consumo}

Definición de formas de cocción relacionadas como factor de riesgo o protector. Esta sección preguntó de manera específica por la forma usual de cocción en el último año para las carnes y los tubérculos (fritos, asados, a la parrilla o cocidos) $(7,8,11,31)$.

Las secciones segunda y tercera no incorporaron mediciones adicionales en los sujetos, se derivaron exclusivamente de la información científica.
Proceso de variables y análisis estadístico. La traducción de los $\mathrm{R} 24 \mathrm{H}$ a nutrientes se realizó utilizando FoodCalc, con base en la tabla de composición de alimentos suministrada por el Instituto Colombiano de Bienestar Familiar y utilizada en la ENSIN-2005 (32).

El procedimiento de selección de alimentos se realizó con Max r en plataforma Windows 98 (26). Para cada alimento seleccionado por nutriente y ciudad, se calcularon el coeficiente $r$ de Person y el $R^{2} w$.

La adecuación de bases de datos para los análisis propuestos se realizó en STATA/SE (33). La descripción de las variables se realizó con estadísticos apropiados, porcentajes para las categóricas y para las continuas, promedios con su intervalo de confianza del 95\% (IC95\%). Para las comparaciones se utilizaron la prueba de ji al cuadrado y la t de Student.

Todos los procedimientos fueron aprobados por los comités de ética del Instituto Nacional de Cancerología y la Universidad Industrial de Santander.

\section{Resultados}

Un total de 422 hombres $(50,1 \%$ y de 420 mujeres (49,9\%) aportaron $906 \mathrm{R} 24 \mathrm{H}, 166$ en Barranquilla, 367 en Bogotá, 125 en Bucaramanga y su área metropolitana, 141 en Cartagena y 107 en Santa Marta. La edad media de los sujetos en Barranquilla fue de 43,4 años (IC95\% 41,9-44,9); en Bogotá, de 43,7 años (IC95\% 42,7-44,6); en Bucaramanga, de 43,9 años (IC95\% 42,2-45,6); en Cartagena, de 44,0 años (IC95\% 42,3-45,7), y en Santa Marta, de 43,0 años (IC95\% 41,2-44,8). Con excepción de Santa Marta $(p=0,043)$, para ninguna ciudad hay diferencia de edad entre hombres y mujeres.

Del total de alimentos consumidos en cada ciudad -mínimo, 189 en Santa Marta; máximo, 429 en Bogotá- y que aportaban a la ingestión de los nutrientes de interés, alrededor de una tercera parte cumplió con los criterios para declararlos elegibles en el proceso de selección. En el cuadro 1 se presenta la información utilizada para la derivación de la lista de chequeo en los cuestionarios de frecuencia de consumo.

Todos los coeficientes $r$ alcanzados para los nutrientes de interés estuvieron por encima de 0,80 -mínimo, 0,82, y máximo, 1,0- El valor mínimo fue para la fibra soluble de la dieta en Santa Marta; el máximo se alcanzó para los diferentes tipos de 
Cuadro 1. Información utilizada para la derivación de los cuestionarios de frecuencia de consumo: número de sujetos, de R24H y de alimentos consumidos y analizados por ciudad.

\begin{tabular}{|c|c|c|c|c|c|c|}
\hline \multirow[b]{2}{*}{ Ciudad } & \multicolumn{2}{|r|}{ Sujetos* } & \multirow{2}{*}{\multicolumn{2}{|c|}{$\mathrm{R} 24 \mathrm{H} \dagger$}} & \multicolumn{2}{|c|}{ Alimentos† } \\
\hline & Total & H $\quad$ M & & & Consumidos & Analizados (\%) \\
\hline Barranquilla $[08 ; 001]$ ‡ & 154 & 78 & 76 & 166 & 261 & $89(34,1)$ \\
\hline Bogotá, D.C. [11; 001] & 344 & 165 & 179 & 367 & 429 & $147(34,3)$ \\
\hline Bucaramanga $[68 ; 001] \mid$ & 115 & 63 & 52 & 125 & 267 & $78(29,2)$ \\
\hline Cartagena $[13 ; 001]$ & 130 & 68 & 62 & 141 & 233 & $72(30,9)$ \\
\hline Santa Marta $[47 ; 001]$ & 99 & 48 & 51 & 107 & 189 & $61(32,3)$ \\
\hline
\end{tabular}

* H. hombre; M: mujer; †: con base en la información recolectada en la Encuesta Nacional de la Situación Nutricional en Colombia, 2005 (23); ¥: Código Departamento Administrativo Nacional de Estadísticas [departamento; municipio]. Incluye del área metropolitana, los códigos 574, 307 y 276 de las ciudades Piedecuesta, Girón y Floridablanca.

grasa, la vitamina $A$ y la fibra insoluble de la dieta en todas las ciudades. El $R^{2} w$ fue igualmente alto para todos los nutrientes y ciudades -mínimo, 47,3, y máximo, 100,0-; el menor valor se obtuvo para la fibra soluble de la dieta en Santa Marta y el máximo, para la fibra insoluble de la dieta en todas las ciudades. El cuadro 2 muestra en detalle los coeficientes $r$ y $R^{2} w$ alcanzados para cada nutriente y ciudad.

Finalmente, se seleccionaron 51 alimentos, 24 hacen parte de la lista de chequeo de Barranquilla, 22 de la de Bogotá, 25 de la de Bucaramanga, 32 de la de Cartagena y 23 alimentos de la de Santa Marta. Nueve alimentos son comunes a las cinco listas de chequeo: aceite cremoso, azúcar granulada, carne de res, huevo cocido, leche de vaca pasteurizada, pan blanco, papa sin cáscara, yuca y zanahoria cruda. Veintidós alimentos fueron específicos para una ciudad y 24 alimentos aportaron a dos, tres o cuatro ciudades.

Dentro de la primera sección de la lista de chequeo, los alimentos se agruparon en 14 grupos, con el fin de garantizar la posibilidad de comparación de los resultados (9-12) y, además, responder a la lógica básica de "alimento fuente" implícita en la nutrición; 1) carne/pollo, 2) aceites y comidas fritas, $3)$ huevos, 4) granos enteros, 5) granos molidos, refinados y tubérculos, 6) productos lácteos, 7) salsas, 8) postres, dulces, 9) bebidas azucaradas, 10) leguminosas, 11) frutas y jugos de frutas, 12) verduras frescas, 13) otros vegetales cocidos y 14) bebidas alcohólicas.

Diecisiete alimentos fueron clasificados en el grupo cinco, seis en los grupos 6 y 11, cuatro alimentos en los grupos 1 y 10, tres en los grupos 2, 8 y 13, dos en el grupo 12, y un alimento en los grupos 3,7 y 9 . En los grupos de granos enteros y de bebidas alcohólicas, no se seleccionó ninguno. El cuadro 3 presenta la lista de alimentos seleccionados para cada ciudad según el grupo asignado.

En la segunda sección de cada cuestionario de frecuencia de consumo, se incorporaron de manera uniforme los siguientes alimentos: carnes rojas en general, carnes de cualquier tipo, embutidos, hortalizas, frutas, leguminosas, huevos, salvado de trigo, productos horneados integrales, cerveza, azúcar y café $(7,8,11)$. La ingestión regular de estos alimentos ha sido sistemáticamente asociada con la génesis del cáncer y, además, permite discriminar fácilmente a los sujetos para asignarlos en niveles de riesgo $(7,8,11)$.

En la tercera sección del cuestionario de frecuencia de consumo, se indagó por las formas de cocción predominantes para las carnes y los tubérculos, y además, se incorporó una pregunta que hace referencia al número de comidas que se realizan en el día. El apéndice 1 ilustra la estructura de los cuestionarios de frecuencia de consumo que se desarrollaron.

\section{Discusión}

Este trabajo es el resultado de la alianza estratégica entre el Instituto Nacional de Cancerología y la Universidad Industrial de Santander, las dos instituciones que lideran en Colombia, de un lado, la atención y la investigación en cáncer, y del otro, la investigación en epidemiología nutricional.

Los cuestionarios de frecuencia de consumo que se crearon son específicos para cada ciudad al incorporar el patrón de consumo de Barranquilla, Cartagena, Santa Marta, Bogotá y Bucaramanga y su área metropolitana. Para que un alimento o conjunto de alimentos alcance poder discriminatorio en una lista de chequeo, es deseable que expliquen 
Cuadro 2. Valores estadísticos alcanzados en el proceso de selección de alimentos para nutrientes específicos; coeficiente de correlación r de Person y coeficiente R2w o de maximización de la varianza-Max R (26).

\begin{tabular}{|c|c|c|c|c|c|}
\hline \multirow[b]{2}{*}{ Nutriente } & \multicolumn{5}{|c|}{ Ciudad } \\
\hline & Barranquilla & Bogotá & Bucaramanga & Cartagena & Santa Marta \\
\hline Kilocalorías & $0,98(74,0) \dagger$ & $0,94(68,4)$ & $0,95(49,9)$ & $0,99(83,2)$ & $0,98(88,0)$ \\
\hline Proteínas & $0,95(68,1)$ & $0,97(74,6)$ & $0,94(69,1)$ & $0,93(84,9)$ & $0,96(75,9)$ \\
\hline Carbohidratos & $0,98(77,7)$ & $0,97(78,1)$ & $0,97(82,6)$ & $0,99(83,5)$ & $0,98(84,0)$ \\
\hline Grasa total & $1,00(93,5)$ & $1,00(93,7)$ & $1,00(96,2)$ & $1,00(98,4)$ & $1,00(96,8)$ \\
\hline Grasa saturada & $0,99(91,3)$ & $0,99(92,5)$ & $0,99(91,4)$ & $1,00(95,4)$ & $1,00(97,0)$ \\
\hline Grasa monoinsaturada & $0,99(91,5)$ & $0,99(92,7)$ & $1,00(96,7)$ & $1,00(98,3)$ & $1,00(98,1)$ \\
\hline Grasa poliinsaturada & $1,00(96,8)$ & $1,00(98,6)$ & $1,00(97,8)$ & $1,00(99,9)$ & $1,00(98,8)$ \\
\hline Fibra soluble en la dieta & $0,94(68,4)$ & $0,94(68,5)$ & $0,92(54,0)$ & $0,86(52,6)$ & $0,82(47,3)$ \\
\hline Fibra insoluble en la dieta & $0,99(100)$ & $0,99(96,6)$ & $1,00(100)$ & $1,00(100)$ & $0,99(100)$ \\
\hline Hierro & $0,91(45,2)$ & $0,92(52,1)$ & $0,95(65,5)$ & $0,85(58,9)$ & $0,92(60,9)$ \\
\hline Vitamina A & $0,98(88,0)$ & $1,00(92,2)$ & $1,00(90,6)$ & $0,99(96,0)$ & $0,97(90,3)$ \\
\hline Vitamina C & $0,94(75,7)$ & $0,93(71,1)$ & $0,96(82,7)$ & $0,92(76,8)$ & $0,90(74,7)$ \\
\hline Ácido fólico & $0,87(46,4)$ & $0,89(58,2)$ & $0,94(77,3)$ & $0,86(54,7)$ & $0,93(65,1)$ \\
\hline
\end{tabular}

$\dagger: r$ de Pearson (R2w).

en buena medida la variabilidad del nutriente de interés, es decir que en el proceso de selección alcancen $R^{2}$ o $R^{2}$ waltos $(9,10)$. Todos los conjuntos de cinco alimentos seleccionados alcanzan $R^{2}$ superiores a 0,70 ; en consecuencia, las listas de chequeo son exhaustivas al incorporar la variabilidad entre personas.

Las dos secciones añadidas a la lista de chequeo permiten, con base en el conocimiento actual, explorar la relación dieta-cáncer, no sólo desde la perspectiva de la ingestión de nutrientes, sino también, desde la de los hábitos y la cultura alimentaria, elementos deseables en la investigación epidemiológica $(11,34)$. Las listas de chequeo desarrolladas son limitadas en ítems, lo que hace que se requiera poco tiempo para su aplicación y disminuye los costos en la investigación epidemiológica.

Tal vez, la principal limitación en las listas de chequeo desarrolladas, es que los $\mathrm{R} 24 \mathrm{H}$ que dieron origen a éstas en vez de recetas, incorporaron los alimentos como ingredientes de recetas -lo ideal es que primen las preparaciones o recetas-. Lo anterior puede llevar a dilución de la asociación en la relación dieta-cáncer 0 , en el caso de que se establezcan relaciones, a falta de especificidad en las recomendaciones derivadas para la población (9-11).

Para ilustrar esta última situación, supóngase que se establece una relación de protección entre el consumo de tomate cocido y el desarrollo de algún tipo de cáncer, pero que, simultáneamente, se establece una relación de riesgo entre el consumo de espaguetis -en los que para su preparación se usó tomate- y el mismo tipo de cáncer, ¿podría, entonces, recomendarse el consumo de tomate?

Esta situación, que se torna compleja para la intervención en la población, es consecuencia de dos aspectos: el primero, relacionado con las interrelaciones entre alimentos, mezcla de alimentos en recetas y formas de cocción de éstas, y el segundo, de las limitaciones que existen en las tablas de composición de los alimentos disponibles en Colombia. Sólo el desarrollo de la epidemiología nutricional y de la investigación epidemiológica aplicada en el estudio de estas relaciones, presionará el desarrollo de estas tablas. En Colombia hay experiencias regionales del desarrollo de tablas específicas de preparaciones o recetas útiles en la investigación epidemiológica (35).

Análisis de los cuestionarios de frecuencia de consumo para establecer relaciones entre dieta y cáncer. Los cuestionarios de frecuencia de consumo permiten realizar múltiples análisis para establecer la relación dieta-cáncer. A continuación se describen algunos de ellos:

a) La lista de chequeo puede ser analizada estableciendo relaciones bivariadas en tablas de contingencia, entre cada alimento o grupo de alimentos y el evento bajo estudio.

b) La lista de chequeo, luego de traducirse a consumo de energía y nutrientes, permite clasificar a los sujetos en niveles de consumo (terciles o cuartiles) y también de acuerdo con su consumo absoluto, lo que permite establecer relaciones bivariadas en tablas de $2 \times \mathrm{n} 0$ en relaciones multivariadas en modelos de regresión. Las 
Cuadro 3. Alimentos seleccionados para cada ciudad.

\begin{tabular}{|c|c|c|c|c|c|}
\hline \multirow[b]{2}{*}{$\begin{array}{l}\text { Alimento o preparación [grupo de alimentos] } \\
\text { Total alimentos seleccionados }\end{array}$} & \multicolumn{5}{|c|}{ Ciudad* } \\
\hline & $\begin{array}{l}\text { Barranquilla } \\
\qquad 24\end{array}$ & $\begin{array}{l}\text { Bogotá } \\
22\end{array}$ & $\begin{array}{l}\text { Bucaramanga } \\
\qquad 25\end{array}$ & $\begin{array}{l}\text { Cartagena } \\
\quad 32\end{array}$ & $\begin{array}{l}\text { Santa Marta } \\
\quad 23\end{array}$ \\
\hline Aceite cremoso o manteca [2] & $\mathrm{x}$ & $\mathrm{x}$ & $\mathrm{x}$ & $\mathrm{x}$ & $\mathrm{x}$ \\
\hline Aceite de girasol [2] & $\mathrm{x}$ & $\mathrm{x}$ & & & $\mathrm{x}$ \\
\hline Aguacate [12] & & $\mathrm{x}$ & $\mathrm{x}$ & $\mathrm{x}$ & \\
\hline Ají picante [7] & & & & $\mathrm{x}$ & \\
\hline Arepa de maíz blanco [5] & $\mathrm{x}$ & $x$ & & & $x$ \\
\hline Arracacha blanca sin cáscara [5] & & & $\mathrm{x}$ & & \\
\hline Arroz blanco [5] & $\mathrm{x}$ & & $\mathrm{x}$ & $\mathrm{x}$ & $\mathrm{x}$ \\
\hline Arveja verde (cualquier preparación) [10] & & $\mathrm{x}$ & & $\mathrm{x}$ & \\
\hline Azúcar granulada [8] & $\mathrm{x}$ & $\mathrm{x}$ & $\mathrm{x}$ & $\mathrm{x}$ & $\mathrm{x}$ \\
\hline Bollo limpio [5] & & & & $\mathrm{x}$ & \\
\hline Bebida de café en infusión [9] & & & $\mathrm{x}$ & $\mathrm{x}$ & \\
\hline Carne de res [1] & $\mathrm{x}$ & $\mathrm{x}$ & $\mathrm{x}$ & $\mathrm{x}$ & $\mathrm{x}$ \\
\hline Chocolate con azúcar [8] & & $\mathrm{x}$ & & & \\
\hline Costilla de res asada [1] & & & & $\mathrm{x}$ & \\
\hline Espaguetis (plato principal) [5] & $\mathrm{x}$ & & & & \\
\hline Fríjol blanco (cualquier preparación) [10] & & & $\mathrm{x}$ & & \\
\hline Fríjol rojo (cualquier preparación) [10] & & & & & $x$ \\
\hline Guayaba rosada (entera o jugo) [11] & $\mathrm{x}$ & & $\mathrm{x}$ & $\mathrm{x}$ & $\mathrm{x}$ \\
\hline Harina de maíz blanco [5] & & & $\mathrm{x}$ & $\mathrm{x}$ & $\mathrm{x}$ \\
\hline Hígado de res [1] & & $\mathrm{x}$ & & & \\
\hline Huevo cocido [3] & $\mathrm{x}$ & $\mathrm{x}$ & $\mathrm{x}$ & $\mathrm{x}$ & $\mathrm{x}$ \\
\hline Leche de vaca líquida entera hervida [6] & & & $\mathrm{x}$ & & \\
\hline Leche de vaca líquida entera pasteurizada [6] & $\mathrm{x}$ & $\mathrm{x}$ & $\mathrm{x}$ & $\mathrm{x}$ & $\mathrm{x}$ \\
\hline Leche de vaca entera en polvo [6] & & & & $x$ & \\
\hline Lenteja sola (cualquier preparación) [10] & $\mathrm{x}$ & & $\mathrm{x}$ & $\mathrm{x}$ & $\mathrm{x}$ \\
\hline Limón entero o jugo [11] & & $\mathrm{x}$ & $\mathrm{x}$ & & $\mathrm{x}$ \\
\hline Mango entero o jugo [11] & & & & $\mathrm{x}$ & \\
\hline Mayonesa [2] & & & & $\mathrm{x}$ & \\
\hline Naranja entera o jugo [11] & $\mathrm{x}$ & $\mathrm{x}$ & $\mathrm{x}$ & & \\
\hline Ñame sin cáscara, cocido u horneado [5] & & & & $\mathrm{x}$ & \\
\hline Queso campesino o costeño [6] & $\mathrm{x}$ & & & $\mathrm{x}$ & \\
\hline Queso semiblando de leche entera [6] & & & & & $\mathrm{x}$ \\
\hline Queso mozarrella [6] & $\mathrm{x}$ & & & $\mathrm{x}$ & \\
\hline Pan blanco [5] & $\mathrm{x}$ & $\mathrm{x}$ & $\mathrm{x}$ & $\mathrm{x}$ & $\mathrm{x}$ \\
\hline Pan de dulce [5] & $\mathrm{x}$ & & & $\mathrm{x}$ & \\
\hline Pan mogolla [5] & & $\mathrm{x}$ & & & \\
\hline Panela [8] & $\mathrm{x}$ & $\mathrm{x}$ & $\mathrm{x}$ & & $\mathrm{x}$ \\
\hline Papa sin cáscara [5] & $\mathrm{x}$ & $\mathrm{x}$ & $\mathrm{x}$ & $\mathrm{x}$ & $\mathrm{x}$ \\
\hline Papa criolla con cáscara [5] & & $\mathrm{x}$ & $\mathrm{x}$ & & \\
\hline Papaya entera o jugo [11] & & & & $\mathrm{x}$ & \\
\hline Plátano [5] & $\mathrm{x}$ & & $\mathrm{x}$ & & $\mathrm{x}$ \\
\hline Plátano colí, colicero o guineo cocido [5] & $\mathrm{x}$ & & & & $\mathrm{x}$ \\
\hline Plátano hartón maduro [5] & & & & $\mathrm{x}$ & \\
\hline Plátano hartón verde [5] & & & & $\mathrm{x}$ & \\
\hline Pechuga de pollo [1] & & & & $\mathrm{x}$ & \\
\hline Remolacha cocida [13] & $\mathrm{x}$ & & & & \\
\hline Tomate chonto cocido [13] & $\mathrm{x}$ & & $\mathrm{x}$ & $\mathrm{x}$ & $\mathrm{x}$ \\
\hline Tomate de árbol entero o cocido [11] & $x$ & & $x$ & $x$ & $x$ \\
\hline Yuca [5] & $\mathrm{x}$ & $\mathrm{x}$ & $\mathrm{x}$ & $\mathrm{x}$ & $\mathrm{x}$ \\
\hline Zanahoria cocida [13] & $\mathrm{x}$ & $\mathrm{x}$ & $x$ & $\mathrm{x}$ & $\mathrm{x}$ \\
\hline Zanahoria cruda [12] & & $\mathrm{x}$ & & & \\
\hline
\end{tabular}

exploraciones deben realizarse con la ingestión absoluta y ajustada por cada 1.000 kilocalorías consumidas (36).

c) Para cada nutriente es posible establecer su consumo a partir del total de la lista de chequeo o con los alimentos específicos que contribuyen a su ingestión en cada ciudad. El análisis de nutrientes que utiliza toda la lista de chequeo, puede llevar a una sobrestimación y a distorsiones en la relación entre dieta y cáncer (14). Las listas de alimentos que contribuyeron a la ingestión específica de cada nutriente y sus porciones por ciudad, son extensas y no se presentan, pero pueden solicitarse a los autores. El análisis con los nutrientes utilizando 
los alimentos específicos que contribuyen a su ingestión, se debe realizar como en b).

d) Es posible agregar el consumo de energía y nutrientes por cada uno de los trece grupos de alimentos finalmente establecidos para, luego, clasificar a los sujetos en niveles de consumo y también de acuerdo con su consumo, lo que permitirá establecer, como en b), relaciones bivariadas en tablas de $2 \times n$ o relaciones multivariadas en modelos de regresión.

e) La lista de chequeo también puede analizarse para cada ítem, estableciendo en ellos frecuencias de consumo referidas a un periodo específico. Para lo anterior, sólo es necesario utilizar amplificadores o divisores en las categorías de frecuencia de consumo que permitan calcular, para cada alimento y sujeto, el consumo medio en un período establecido. Los cuestionarios de frecuencia de consumo que se desarrollaron hacen alusión al consumo del último año, pero es posible cambiar esta unidad en los resultados a otras como día, semana, mes, etc.

f) Con la lista de chequeo es posible establecer diversos índices al poner en operación las variables "alimento", "grupo de alimentos", "frecuencia de consumo", e "ingestión estimada".

g) Recientemente, se ha reportado el análisis de un cuestionario de frecuencia de consumo desarrollado mediante análisis de factores y componentes principales con los ítems de la lista de chequeo, tipologías de consumo o patrones de consumo (37). Esta novedosa perspectiva permite establecer patrones de ingestión que, en teoría, incorporan la interacción entre diferentes tipos de nutrientes y alimentos, una de las principales limitaciones en los análisis de la dieta en estudios epidemiológicos. Con los cuestionarios de frecuencia de consumo que se desarrollaron es posible, mediante análisis de factores, componentes principales o análisis RASCH (38), establecer tipologías tanto con los ítems de alimentos como con los grupos establecidos.

h) La segunda sección del cuestionario de frecuencia de consumo permite un análisis bivariado entre cada alimento y el evento de interés en tablas de $2 \times 2$, y además, agrupar alimentos para generar nuevas categorías de consumo, de acuerdo con las hipótesis del investigador o los resultados previos obtenidos del análisis de la lista de chequeo.

i) Finalmente, la tercera sección permite establecer, en relaciones bivariadas, el grado de asociación del método de cocción predominante en las carnes rojas, blancas y los tubérculos, con el desarrollo de cáncer.

Implicación de los resultados. La disponibilidad de datos de consumo en la ENSIN-2005 y la futura ENSIN-2010, permitiráel desarrollo decuestionarios de frecuencia de consumo para otras ciudades colombianas que, como las cinco inicialmente contempladas, contribuyen significativamente a la incidencia y mortalidad por cáncer (3-6).

Los cuestionarios de frecuencia de consumo que se desarrollaron pueden utilizarse desde ya en la investigación epidemiológica aplicada, con la obligatoria consideración de que los resultados, para ser válidos, deben calibrarse con base en los estudios de reproducibilidad y validez que están en marcha en el país (14-22).

Las alternativas de análisis descritas son algunas de las posibles con los cuestionarios de frecuencia de consumo que se desarrollaron. Estos cuestionarios de frecuencia de consumo difieren substancialmente de otros utilizados en países desarrollados. La aparente simplicidad de los aquí desarrollados se debe a los métodos computacionales y estadísticos utilizados, y la homogeneidad en la dieta colombiana (30). La ingestión en la dieta es una de las variables que en el complejo causal del cáncer puede actuar como variable de explicación o de confusión dentro de modelos multivariados.

Los cuestionarios de frecuencia de consumo que se desarrollaron permiten profundizar en el estudio de las interrelaciones entre alimentos, nutrientes, hábitos y preparación de alimentos, y su papel en la génesis del cáncer en Colombia. Este trabajo debe entenderse como progresivo y complementario, e incluirá el desarrollo de otros cuestionarios de frecuencia de consumo para ciudades colombianas donde el cáncer es igualmente importante dentro del perfil epidemiológico.

\section{Agradecimientos}

Al Instituto Colombiano de Bienestar Familiar por permitir el uso de las bases del consumo dietario de la ENSIN-2005 (23).

\section{Conflicto de intereses}

Los autores declaramos que no existe conflicto de intereses en este manuscrito.

\section{Financiación}

Este estudio fue cofinanciado por la Universidad Industrial de Santander a través del Centro de 
Investigaciones Epidemiológicas (CIE), la Escuela de Nutrición y Dietética y el grupo de investigación "Observatorio Epidemiológico de Enfermedades Cardiovasculares, COL0014153", y el Instituto Nacional de Cancerología, Empresa Social del Estado, a través del grupo de investigación "Salud Pública y Epidemiología del Cáncer, COL0001275”.

\section{Referencias}

1. Bautista L, Orostegui M, Vera LM, Prada GE, Orozco LC, Herrán OF. Prevalence and impact of major cardiovascular risk factors in Bucaramanga, Colombia. Results from the CARMEN baseline survey. Eur J Cardiovasc Prev Rehabil. 2006;5:769-75.

2. Ministerio de la Protección Social, Organización Panamericana de la Salud. Situación de Salud en Colombia: indicadores básicos 2007. Bogotá, D.C.: Ministerio de la Protección Social; 2008.

3. Ochoa FL, Montoya LP. Carga de cáncer en Colombia Rev Colomb Cacerol. 2007;11:168-73.

4. Ochoa FL, Montoya LP. Mortalidad por cáncer en Colombia Cuando aumentar no es mejorar. Rev CES Med. 2003;17:7-22.

5. Piñeros M, Murillo R. Incidencia de cáncer en Colombia: importancia de las fuentes de información en la obtención de cifras estimativas. Rev Colomb Cancerol. 2004;8:5-14.

6. Murillo R, Piñeros M, Hernández G. Atlas de mortalidad por cáncer en Colombia. Bogotá D.C.: Imprenta Nacional de Colombia; 2003.

7. Organización Mundial de la Salud. Food, nutrition and the prevention of cancer: A global perspective, 2003 Publicación científica y técnica No. 583. Washington D.C.: Organización Mundial de la Salud; 2003.

8. Organización Mundial de la Salud. Dieta, nutrición y prevención de enfermedades crónicas, 2003. Publicación científica y técnica No. 916. Washington D.C.: Organización Mundial de la Salud; 2003.

9. Willet WC. Nutritional epidemiology. 2 edition. New York: Oxford University Press; 1998.

10. Margetts BM, Nelson M. Design concepts in nutritional epidemiology. 2 edition. New York: Oxford University Press; 1996.

11. Bowman BA, Russell RM. Conocimientos actuales sobre nutrición. 8 edición. Publicación científica y técnica número 592. Washington D.C.: Organización Mundial de la Salud; 2003.

12. Bautista L, Herrán OF, Pryer JA. Development and simulated validation of a food-frequency questionnaire for the Colombian population. Public Health Nutr. 2005:8:181-8.

13. Herrán OF, Ardila MF. Validity and reproducibility of two semi-quantitative Alcohol frequency questionnaires for Colombian population. Public Health Nutr. 2006;9:763-70.

14. Kipnis V, Freedman LS. Impact of exposure measurement error in nutritional epidemiology. J Natl Cancer Inst. 2008;100:1658-9.

15. Kaaks R, Riboli E, van Staveren W. Calibration of dietary intake measurements in prospective cohort studies. Am J Epidemiol. 1995;142:548-56.
16. Rosner B, Willett WC, Spiegelman DN. Correction of logistic regression relative risk estimates and confidence intervals for systematic with in-person measurement error. Stat Med. 1989;8:1051-69.

17. Rosner B, Spiegelman D, Willett WC. Correction of logistic regression relative risk estimates and confidence intervals for measurement error: the case of multiple covariates measured with error. Am J Epidemiol. 1990;132:734-45.

18. Kristal AR, Peters U, Potter JD. Is it time to abandon the food frequency questionnaire? Cancer Epidemiol Biomarkers Prev. 2005;14:2826-8.

19. Freudenheim JL, Marshall JR. The problem of profound mismeasurement and the power of epidemiologic studies of diet and cancer. Nutr Cancer. 1988;11:243-50.

20. Kipnis V, Midthune D, Freedman LS, Bingham S, Schatzkin A, Subar A, et al. Empirical evidence of correlated biases in dietary assessment instruments and its implications. Am J Epidemiol. 2001;153:394-403.

21. Kaaks R, Ferrari P, Ciampi A, Plummer M, Riboli E. Uses and limitations of statistical accounting for random error correlations, in the validation of dietary questionnaire assessments. Public Health Nutr. 2002;5:969-76.

22. Tooze JA, Midthune D, Dodd KW, Freedman LS, KrebsSmith SM, Subar AF, et al. A new statistical method for estimating the usual intake of episodically consumed foods with application to their distribution. J Am Diet Assoc. 2006;106:1575-87.

23. Instituto Colombiano de Bienestar Familiar. Encuesta Nacional de la Situación Nutricional en Colombia, 2005. Bogotá D.C.: ICBF; 2005.

24. Ochoa FL, Montoya LP. Mortalidad por cáncer en Colombia 2001. Rev CES Med. 2004;18:19-36.

25. Mark SD, Thomas DG, Decarli A. Measurement of exposure to nutrients: an approach to the selection of informative foods. Am J Epidemiol. 1996;143:514-21.

26. Thomas DG. Software Max r 2.1. Bethesda: National Cancer Institute; 1996.

27. Stryker WS, Salvini S, Stampfer MJ, Sampson L, Colditz GA, Willett WC. Contributions of specific foods to absolute intake and between-person variation of nutrient consumption. J Am Diet Assoc. 1991;91:172-8.

28. Decarli A, Ferrarroni M, Palli D. A reduced questionnaire to investigate the Mediterranean diet in epidemiologic studies. Epidemiology. 1994;5:251-6.

29. Herrán OF, Gamboa EM, Prada GE. Diseño y eficacia de pruebas para determinar la deficiencia de hierro. Rev Chil Nutr. 2006;33:518-26.

30. Herrán OF, Quintero DC, Ardila MF. Sources and magnitude of the variation in the diet of Bucaramanga, Colombia. Rev Chil Nutr. 2006;33:55-64

31. Willett WC. Diet and cancer: One view at the start of the millennium. Cancer Epidemiol Biomarkers Prev. 2001;10:3-8.

32. Lauritsen J. Software FoodCalc v. 1.3. Diet, cancer and health project. Copenhague: Danish Cancer Society; 1998.

33. Stata Corporation. Software STATA/SE v. 10 (StataCorp. 2007. Stata Statistical Sofware: Release 10. College Station, TX: Stata Corporation). Texas: Stata Press; 2007. 
34. Fritsch HM, Salgado-Martínez H. Manual de encuestas de dieta. Perspectivas en salud pública, No. 23. Cuernavaca: Instituto Nacional de Salud Pública; 1996.

35. Herrán OF, Bautista LE, Quintero DC. Tabla de composición de alimentos consumidos en Bucaramanga. Segunda edición. Bucaramanga: Universidad Industrial de Santander; 2003.

36. Hansen Gaurth R, Wyse BW. Expression of nutrient allowances per 1,000 kilocalories. J Am Diet Assoc. 1980;76:223-7.
37. Williams CD, Satia JA, Adair LS, Stevens J, Galanko J, Keku TO, et al. Dietary patterns, food groups, and rectal cancer risk in whites and African-Americans. Cancer Epidemiol Biomarkers Prev. 2009;18:1552-61.

38. Bond TG, Fox CM. Applying the Rasch Model: Fundamental measurement in the human sciences. 2 edition. London: Lawrence Erlbaum Associates Publishers; 2007.

\section{Apéndice 1.}

Formato para chequeo de frecuencia de consumo de alimentos

¡Por favor!, no deje espacios en blanco

\section{Primera sección}

¿Con qué frecuencia come los siguientes alimentos? (en el último año)

\begin{tabular}{|c|c|c|c|c|c|c|c|c|c|}
\hline $\begin{array}{l}\text { Alimento } \\
\text { o preparación }\end{array}$ & $\begin{array}{c}1 \\
2 \text { o más } \\
\text { veces al día }\end{array}$ & $\begin{array}{c}2 \\
1 \text { vez } \\
\text { al día } \\
\text { semana }\end{array}$ & $\begin{array}{c}3 \\
2 \circ 3 \\
\text { veces por } \\
\text { semana }\end{array}$ & $\begin{array}{c}4 \\
4 \circ 5 \\
\text { veces por }\end{array}$ & $\begin{array}{c}\mathbf{5} \\
1 \text { vez } \\
\text { por semana } \\
\text { mes }\end{array}$ & $\begin{array}{c}6 \\
1 \text { a } 3 \\
\text { veces por } \\
\text { año }\end{array}$ & $\begin{array}{c}7 \\
5 \text { a } 10 \\
\text { veces por } \\
\text { año }\end{array}$ & $\begin{array}{c}8 \\
1 \text { a } 4 \\
\text { veces por }\end{array}$ & $\begin{array}{c}9 \\
\text { Nunca }\end{array}$ \\
\hline \multicolumn{10}{|c|}{ Grupo 1. Carne/pollo } \\
\hline $\begin{array}{l}301 \text { Carne de res } \\
\text { (cualquier corte) }\end{array}$ & 1 & 2 & 3 & 4 & 5 & 6 & 7 & 8 & 9 \\
\hline
\end{tabular}

Segunda sección

¿Con qué frecuencia come los siguientes alimentos o preparaciones? (en el último año)

\begin{tabular}{|c|c|c|c|c|c|c|c|c|c|}
\hline & 1 & 2 & 3 & 4 & 5 & 6 & 7 & 8 & 9 \\
\hline $\begin{array}{l}\text { Alimento } \\
\text { o preparación }\end{array}$ & $\begin{array}{c}2 \text { o más } \\
\text { veces al día }\end{array}$ & $\begin{array}{c}1 \text { vez } \\
\text { al día } \\
\text { mes }\end{array}$ & $\begin{array}{c}203 \\
\text { veces por } \\
\text { semana }\end{array}$ & $\begin{array}{l}405 \\
\text { veces por } \\
\text { semana }\end{array}$ & $\begin{array}{c}1 \mathrm{vez} \\
\text { por semana }\end{array}$ & $\begin{array}{c}1 \text { a } 3 \\
\text { veces por } \\
\text { mes }\end{array}$ & $\begin{array}{l}5 \text { a } 10 \\
\text { veces por } \\
\text { año }\end{array}$ & $\begin{array}{c}1 \text { a } 4 \\
\text { veces por } \\
\text { año }\end{array}$ & Nunca \\
\hline $\begin{array}{l}\text { Carnes rojas } \\
\text { en general }\end{array}$ & 1 & 2 & 3 & 4 & 5 & 6 & 7 & 8 & 9 \\
\hline
\end{tabular}

\section{Tercera sección}

(Las siguientes preguntas son relativas al comportamiento durante el último año.)

¿Cuántas comidas realiza usted, en promedio, al día?

¿Cuál es la forma de cocción que ha predominado, la más común, en que usted consume los siguientes alimentos?

Carnes rojas:

Fritas

Asadas

A la parrilla (carbón o leña) $\square 3$

Cocido o vapor 